Objective This systematic review was performed to summarise RCTs assessing the efficacy and safety of ginseng treatment in the Korean literature.

Method The study involved systematic searches conducted in eight Korean Medical databases. The methodological quality of all of the included studies was assessed using the Cochrane ROB tool. We included all RCTs on any type of ginseng compared to placebo, active treatment or no treatment in healthy individuals or patients regardless of conditions.

Results In total, 30 randomised clinical trials were included. Nine RCTs assessed the effects of ginseng on exercise capacity, cognitive performance, somatic symptoms, quality of life, and sleeping in healthy persons. Six RCTs tested ginseng compared with placebo for erectile dysfunction, while another four studies evaluated the effects of ginseng against no treatment for gastric and colon cancer. Two RCTs compared the effect of red ginseng on diabetes mellitus with no treatment or placebo, and the other nine RCTs assessed the effects of ginseng compared with placebo or no treatment on various conditions. However, the 20 newly added trials may provide useful information for future trials.

Discussion Most RCTs published in the Korean literature have not been included in up-to-date systematic reviews. Although the quality of RCTs published in the Korean literature was generally poor, this review is useful for researchers to access studies that were originally published in languages that they would otherwise be unable to read and due to the paucity of evidence on this subject.

\section{P023 OBSERVATIONAL DRUG THERAPY TRIALS ADD UP USEFUL INFORMATION AS COMPARED WITH RANDOMISED CONTROLLED TRIALS: CASE MULTIPLE SCLEROSIS}

S Atula, L Lodenius, J Komulainen. Finnish Medical Society Duodecim, Current Care, Helsinki, Finland

\section{0:1136/bmjqs-2013-002293.132}

Background Clinical practice guidelines (CPG's) are predominantly based on randomised controlled trials (RCT's). However, the number of published observational, non-randomised trials is high and yet, the information they contain is often excluded while compiling CPG's.

Objectives We compared randomised and observational clinical treatment trials, published within one year, using multiple sclerosis (MS) as a model. The aim was to find out whether valuable information for CPG's can be discovered by using observational studies.

Methods We collected all publications of MS treatment in Medline during year 2012, using Scottish Intercollegiate Guidelines Network (SIGN) search filtres for randomised and observational trials for making a systematic literature review. The clinical drug trials on adults with MS, published in English were included. Case reports and symptomatic treatment trials were excluded.

Results A total of 24 RCT's and 45 observational trials were found. The median number of participants in RCT's was 430 (range 66 to 2244) and in observational trials 118 (range 5 to 22255). Twenty RCT's were efficacy trials, 2 evaluated health economics and one each safety and pharmacology. Twenty-eight observational trials had efficacy as a primary end-point, 10 addressed safety, 5 treatment adherence and one each pharmacology and health economics.
Discussion Most trials addressing safety or compliance issues were observational, therefore important safety and adherence data could be lost by omitting them.

Implications for Guideline Developers/Users Observational trials should be considered while compiling CPG's.

\section{P030 E-TOOL FOR PATIENTS WITH NON-HODGKIN'S LYMPHOMA TO IMPROVE GUIDELINE ADHERENCE}

${ }^{1} \mathrm{~J}$ Stienen, ${ }^{1} \mathrm{R}$ Hermens, ${ }^{2} \mathrm{H}$ van Krieken, ${ }^{3} \mathrm{~S}$ van de Schans, ${ }^{4} \mathrm{H}$ Dekker, ${ }^{5} \mathrm{R}$ van der Maazen, ${ }^{1} \mathrm{~L}$ Wennekes, ${ }^{6} \mathrm{~N}$ Blijlevens, ${ }^{7} \mathrm{~N}$ Ottevanger. ${ }^{1}$ Scientific Institute for Quality of Healthcare, Radboud University Medical Centre, Nijmegen, Netherlands; '2Department of Pathology, Radboud University Nijmegen Medical Centre, Nijmegen, Netherlands, ${ }^{3}$ Department of Cancer Registry and Research, Comprehensive Cancer Centre the Neth, Utrecht, Netherlands; ${ }^{4}$ Department of Radiology, Radboud University Nijmegen Medical Centre, Nijmegen, Netherlands, ${ }^{5}$ Department of Radiotherapy, Radboud University Nijmegen Medical Centre, Nijmegen, Netherlands; ${ }^{6}$ Department of Hematology, Radboud University Nijmegen Medical Centre Nijmegen Netherlands ${ }^{7}$ Department of Medical Oncology, Radboud University Nijmegen Medical Centre Nijmegen Netherlands

\section{0:1136/bmjqs-2013-002293.133}

Background Patient education should be part of improvement strategies to increase guideline adherence by professionals.

Objectives This study evaluates an e-tool designed in response to patients' need for more complete information regarding diagnostics, therapy and after-care (based on previous research). The e-tool aims to inform patients about non-Hodgkin's lymphoma (NHL)-care and gives patients the possibility to check their own care pathway and register personal experiences.

Methods The e-tool was developed in consultation with the Dutch Lymphoma Patients Organization and evaluated by NHLpatients, professionals and laymen. Feedback was asked concerning lay-out, user convenience, information provision and general strengths and weaknesses of the e-tool. The effect of the e-tool is now tested in 9 Dutch hospitals. Patients are included from November 2012 to November 2013. All patients receive patient information and an informed consent form. The process of inclusion is continuously monitored.

Results In the development phase, 18 out of 26 feedback forms were filled out. Information needs were satisfactory and clearness of navigation and information on new therapies were improved after feedback. In the first 3 months of the testing phase, 25 of the historically estimated 50 patients received patient information, 12 consented and 8 used the e-tool.

Discussion The e-tool seems feasible to empower patients regarding their NHL-care pathway. However, distribution of patient information is not yet optimal. Patients' experiences with the e-tool and possible effects on quality of care is tested in a randomised controlled trial.

Implications Educational e-tools for patients may help to improve guideline development and adherence.

\section{P038 COMPLETING THE PDCA CIRCLE FOR GUIDELINES WITHIN ONE ORGANISATION}

${ }^{1} \mathrm{D}$ Stemkens, ${ }^{1} \mathrm{~K}$ Aben, ${ }^{2} \mathrm{~S}$ Osanto, ${ }^{1} \mathrm{~J}$ de Groot, ${ }^{1} \mathrm{E}$ Verhoof. ${ }^{1} \mathrm{KKNL}$, Comprehensive Cancer Centre The Netherlands, Utrecht, Netherlands; ${ }^{2}$ Leiden University Medical Center, Leiden, Netherlands

\section{0:1136/bmjqs-2013-002293.134}

Background Our organisation is closely involved in the continuous process of developing, implementing and evaluating 